\title{
Introduction to Working with Military Families
}

\author{
Adrian Blow ${ }^{1}$
}

Published online: 9 August 2015

(C) Springer Science+Business Media New York 2015

After the 9/11/2001 terrorist attacks in New York, the United States engaged in the global war on terror with two wars, primarily in Iraq and Afghanistan, leading to sustained military operations that lasted for over a decade. Service members deployed to dangerous combat locations, and their families who remained home juggled the numerous demands of daily life. The deployment/s of a service member and subsequent reintegration represents a period of sustained stress for these families and everyone in the system is affected, sometimes negatively, and personal and family change and growth always result from the experience.

There has been a large and growing body of literature related to families and military deployment, especially over the last decade. Deployment is an essential and noble component of life in the military. Service members risk their lives and make great sacrifices to defend the freedoms of citizens at home. Yet, deployment and related events represent a crisis for members of families who face many changes and transitions during this time. Post deployment, family members are faced with getting to know each other again, a process made more difficult if the deployment was difficult and family life now includes variables such as poor mental health, financial strain, or recovery from injury. These changes, no matter how big or small, represent losses and additions to daily life and require many skills and qualities including adaptability, communication, patience, understanding, conflict resolution, anxiety

Adrian Blow

blowa@msu.edu

1 Couple and Family Therapy Program, Michigan State University, 552 W Circle Drive, East Lansing, MI 48824 , USA management, boundary negotiation, co-parenting, building connection, and acceptance, to name just a few.

Effective interventions for military families have the potential to result in changes that have long-lasting consequences across generations. From a military perspective, service members are more content and able to focus more easily on their work when things are going well at home. From a family perspective, children are better off when parents get along, for example, when they are communicating well, agree on family rules and routines, and show positive examples of love. From an individual perspective, life is easier to cope with when things run smoothly on the home front. When relationships are working well, family is the best support system, the finest motivator, and the most prized team. Positive family support makes it easier to face mental health concerns, employment difficulties, and the aftermath of war. Conversely, negative family relationships add to stress, exacerbate mental health problems, and can lead to detrimental outcomes including suicide, interpersonal violence, and other regrettable life-altering decisions.

In this special issue focused on military families, twelve articles, authored by a diverse array of scholars, provide insight into the wellbeing of these families, and interventions to strengthen family functioning. Two articles focus on helping children who are dealing with the deployment of a parent in the military. Two articles describe prevention approaches for couples and interventions aimed at educating couples before, during, and after deployment, in a way that addresses problems before they even start. One paper focuses on an intervention for military spouses, a group who have needs for added supports and understanding during a difficult time. Four papers describe different interventions for couples including those dealing with PTSD, intimate partner violence, and sexual trauma. One of these papers includes an innovative description on 
how to intervene with couples who are separated by distance from each other and the therapist, using technology. Three papers describe research studies on military couples including their biopsychosocial health, dual military couples, and the important role of technology during a deployment. It is hoped that these articles will lead to more effective intervention deliveries to these families, and will spark ideas for future research. 